\title{
Discurso colonial en videojuegos serios y posibilidades para ecolonialidad en terceros espacios
}

\section{Sección: Dossier \\ Recibido: 30/08/2021 \\ Aceptado: $21 / 11 / 2021$}

\section{Colonial Discourse in Serious Games and Possibilities for Decoloniality in Third Spaces}

\author{
Amanda Rodríguez Espínola \\ Universidad de Colorado Boulder, Estados Unidos \\ ORCID: https://orcid.org/0000-0002-5676-9770 \\ amanda.rodriguezespinola@gmail.com
}

\begin{abstract}
Resumen
Organizaciones internacionales han encargado la producción de videojuegos para crear conciencia sobre el desarrollo económico y los problemas humanitarios, estableciendo redes basadas en la empatía intergrupal y las diversas realidades que enfrentan los jugadores. Sin embargo, existe una brecha en el estudio de los juegos digitales como herramientas que pueden mejorar la empatía entre grupos y las implicaciones del discurso que tienen estos juegos sobre la otredad. El propósito de este artículo es explorar las formas en que los videojuegos construyen nociones de alteridad arraigadas en el pensamiento colonial, posicionando a los desarrolladores y jugadores como reproductores de esta retórica. Este artículo explorará el concepto de empatía y cómo los videojuegos serios pueden usarse como herramientas para fomentarla. Luego, se usa el juego $E V O K E$ para ejemplificar este género y resaltar sus limitaciones. Finalmente, se ofrece una alternativa para fomentar la empatía a través de videojuegos serios dentro de un proyecto decolonial. La importancia del proyecto radica en reunir los campos del estudio de juegos y del cambio social a través de la empatía y proponer una lente decolonial a través de la cual reconocer las relaciones problemáticas entre los juegos para el cambio y la empatía.
\end{abstract}

Palabras clave: juegos serios, teoría decolonial, colonialismo, empatía, videojuegos.

\begin{abstract}
International organizations have commissioned videogame production to raise awareness of economic development and humanitarian issues, establishing networks based on intergroup empathy and the various realities players face. However, there is a gap in the study of digital games as tools that can improve empathy between groups and the implications of these games' discourse on otherness. The purpose of this article is to explore how video games construct such notions rooted in colonial thought. Thus, game developers and players are reproducing colonialist rhetorics. This article explores the concept of empathy and how serious games can be used as tools to foster it. The game EVOKE is then used to exemplify this genre and highlight its limitations. Finally, an alternative is offered to promote empathy through serious games within a decolonial project. The importance of the project lies in bringing together the fields of game studies and social change through the exploration of empathy building and proposing a decolonial lens through which to acknowledge problematic relations between games for change and empathy.
\end{abstract}

Keywords: serious games, decolonial theory, colonialism, empathy, videogames.

\section{Introducción}

Con el desarrollo de las tecnologías de la comunicación, los medios de comunicación se convirtieron en una importante fuente de información capaz de llegar a grandes audiencias. La segunda mitad del siglo XX vio el surgimiento del edutainment como un campo académico con el propósito de "diseñar e implementar un mensaje mediático para entretener y educar, con el fin de aumentar el conocimiento de la audiencia sobre un tema educativo [y] contribuir a 
cambio social". ${ }^{1}$ A medida que los medios digitales aumentan su impacto en una sociedad hipermediatizada, los videojuegos digitales han adquirido relevancia no solo como objetos culturales, sino como herramientas con fines educativos y de entretenimiento. Algunas investigaciones han señalado las diversas formas en que se pueden estudiar los juegos digitales en relación con campos académicos como educación (Wang y Singhal, 2009), estudios religiosos (Campbell et al., 2016) y salud pública (Millington, 2016). Para comprender cómo los juegos digitales brindan al usuario un espacio de aprendizaje, primero es necesario comprender cómo, al ser medios de comunicación, se han convertido en parte de los procesos sociales e institucionales.

De acuerdo con la teoría de la mediatización (Hjarvard, 2011), la sociedad contemporánea "está impregnada de los medios de comunicación, hasta el punto de que los medios de comunicación ya no pueden ser concebidos como separados de las instituciones culturales y sociales". ${ }^{2}$ Es decir, los medios de comunicación ya no deberían considerarse separados de los procesos sociales y culturales, sino como una parte intrínseca de ellos. Los videojuegos se han convertido en un medio e industria cultural poderosa y no deberían ser excluidos del discurso de la mediatización. Ocupan una "posición dominante como proveedores de productos y creencias culturales"3 que se extiende a las estrategias de desarrollo económico establecidas por organizaciones internacionales como la Organización de las Naciones Unidas y el Banco Mundial.

El continuo desarrollo de las tecnologías de la información y la comunicación (TIC) ha dado como resultado un "clima favorable para el uso de juegos digitales para la enseñanza, el aprendizaje y la formación, [ellos] pueden resultar una herramienta de aprendizaje activo crucial y relevante para involucrar a una generación de estudiantes criado en medios interactivos y participativos". ${ }^{4}$ Durante las últimas dos décadas, las Naciones Unidas y el Banco Mundial han empleado videojuegos para crear conciencia sobre cuestiones humanitarias y de desarrollo económico. Originalmente, estos juegos estaban dirigidos a usuarios de Europa y Estados Unidos. Pero, en los últimos años, el movimiento de los videojuegos serios ha impulsado una plataforma más inclusiva que incluya a jugadores en países como Kenia y Tanzania (Fisher, 2016).

La literatura ha reconocido el potencial de los juegos digitales como mecanismo para desarrollar comportamientos empáticos entre los usuarios (Belman y Flanagan, 2010; Darvasi, 2016; Hasler et al., 2014; Singhal, 2013). Si bien existe un creciente interés en el uso de juegos digitales como herramientas para fomentar la empatía entorno a los problemas sociales, existe una brecha de investigación en el estudio de las "implicaciones políticas económicas de la producción de DDG [juegos digitales para el desarrollo económico, por sus siglas en inglés]". Este estudio parte de la premisa de que los juegos digitales en general, y los videojuegos serios

\footnotetext{
1 "[D]esigning and implementing a media message to both entertain and educate, in order to increase audience knowledge about an educational issue [and] contribute to social change" (Singhal y Rogers, 1999, p. xii). Salvo que se indique lo contrario todas las traducciones son de la autora.

2 "[C]ontemporary society is permeated by the media, to an extent that the media may no longer be conceived of as separate from cultural and other social institutions" (Hjarvard, 2008, p. 105).

3 "[D]ominant position as providers of cultural products and beliefs" (Hjarvard, 2008, p. 108).

4 "[F]avorable climate for the use of digital games for teaching, learning and training, [and they] may prove a crucial and relevant active learning tool to engage a generation of students reared on interactive and participatory media" (Darvasi, 2016, p. 3).

5 "[P]olitical economic implications of the production of DDG [digital development games]" (Fisher, 2016, p. 33).
} 
en particular, basados en su potencial único para facilitar los procesos necesarios para generar empatía intergrupal, pueden operar como una herramienta importante para las organizaciones internacionales que llevan a cabo programas de cambio social. Siguiendo esta premisa, este estudio hace una crítica a la retórica colonial incorporada en algunos videojuegos serios con fines de cambio social y sugiere un enfoque alternativo como proyecto para decolonizar la narrativa y mecánica de los juegos serios. La importancia de reconocer la reproducción del pensamiento colonial en los videojuegos como una problemática seria se basa en la creencia de Foucault de que existe una estrecha relación entre la producción de poder y la producción de conocimiento (Foucault y Gordon, 1980; Mills, 2013). Esto, traducido a la industria del videojuego, significa que "aquellos que hacen juegos de computadora estrechan las culturas y las inscriben con una retórica de influencia colonialista, entonces los creadores de juegos y los jugadores están replicando el proyecto colonialista". 6

Este artículo acerca los campos del estudio de juegos y el cambio social a través de la exploración de la construcción de la empatía, uno de los objetivos primarios en la implementación de estrategias de cambio social y de desarrollo económico, en un contexto que impulsa la decolonización de los videojuegos serios. En última instancia, el objetivo es proporcionar un espacio donde ambos campos puedan ser reconocidos por el otro y crear asociaciones que aumenten su visibilidad y, con suerte, la implementación de proyectos en entornos reflexivos.

\section{Empatía y videojuegos. Videojuegos serios}

Los videojuegos que están diseñados con fines educativos y de aprendizaje se han categorizado como videojuegos serios. Aunque no existe una definición única de lo que estos constituyen (Darvasi, 2016; Marsh, 2011; Sanford et al., 2015), para los propósitos de este artículo, se utiliza la definición de Marsh (2011):

Los juegos serios son juegos digitales, simulaciones, entornos virtuales y medios de realidad mixta que brindan oportunidades para participar en actividades a través de una narrativa/ historia receptiva, jugabilidad o encuentros para informar, influir, para el bienestar y/o la experiencia para transmitir significado. La calidad del éxito de los juegos serios se caracteriza por el grado en que se ha cumplido el propósito. ${ }^{7}$

Ha habido cierto debate sobre la categorización de los videojuegos serios. El argumento principal es el rol del entretenimiento y la diversión en este tipo de juegos y si el entretenimiento minimiza los fines educativos. Otra preocupación que se plantea es que el uso de la palabra 'serio' implica que estos juegos son automáticamente mejores que los juegos no

\footnotetext{
6 " [T] hose making computer games narrow cultures and inscribe them with a colonialist influenced rhetoric, then the game makers and the game players are replicating the colonialist project" (Sheldon, 2008, p. 1).

7 "Serious games are digital games, simulations, virtual environments and mixed reality/media that provide opportunities to engage in activities through responsive narrative/story, gameplay or encounters to inform, influence, for well-being, and/or experience to convey meaning. The quality or success of serious games is characterized by the degree to which purpose has been fulfilled. Serious games are identified along a continuum from games for purpose at one end, through to experiential environments with minimal or no gaming characteristics for experience at the other end" (Marsh, 2001, p. 63).
} 
serios o comerciales. Marsh (2011) sugiere que mientras el propósito tenga cierto grado de éxito para influir, informar, persuadir o transmitir, la cuestión del predominio del entretenimiento no debería tener importancia.

Bogost (2008) ofrece el concepto de procedural rhetoric para capturar la noción de que los videojuegos construyen significado al hacer "afirmaciones sobre el mundo, que los jugadores pueden comprender, evaluar y deliberar". ${ }^{8}$ Considera los procesos adscritos dentro de los sistemas de reglas y el juego como un espacio para explorar las posibilidades que brindan las reglas al manipular sistemas simbólicos (Bogost, 2010). Como tal, el sistema de reglas de un juego implica tomar decisiones para seleccionar algunos símbolos sobre otros, lo que implica una consecuencia. Dado que los videojuegos serios permiten la toma de perspectiva en un entorno inmersivo (Belman y Flanagan, 2010), se han convertido en un tercer espacio en el que el contacto grupal imaginado puede fomentar el diálogo y la cooperación intergrupal, desarrollando un sentido de empatía entre los participantes.

\section{Empatía}

Profesionales y académicos en el campo de la resolución de conflictos y la reconciliación señalan a la empatía como un elemento clave para reducir los prejuicios entre miembros de diferentes grupos y fomentar el cambio social (Calloway-Thomas, 2010; Darvasi, 2016). La empatía se entiende como la "capacidad de ver los problemas y eventos desde la perspectiva de las personas al otro lado de una disputa". ${ }^{9}$ Por tanto, la falta de empatía es "una de las barreras para la resolución de conflictos y la reconciliación". ${ }^{10}$

La empatía puede fomentar la comprensión y la cooperación entre grupos, lo que les permite alcanzar un objetivo común, aumenta la disposición a aceptar acuerdos que involucren a ambos grupos, mejora la cooperación y la coexistencia, así como el desarrollo un cierto nivel de confianza entre los miembros de grupo opuesto (Malhotra y Liyanage, 2005). Según Belman y Flanagan (2010), la empatía se puede clasificar en dos grandes grupos: 1) empatía cognitiva, que requiere que uno ocupe voluntariamente el lugar o el punto de vista del otro; y 2) empatía emocional, que puede referirse a la experiencia indirecta del estado emocional de otra persona (empatía paralela), o una respuesta emocional diferente a la que la otra persona está experimentando (empatía reactiva). Los procesos a través de los cuales se obtiene la empatía se conocen como empatía disposicional e inducida. La primera se refiere a los niveles existentes de voluntad y capacidad de un individuo para sentir empatía y cómo afectan las actitudes y el comportamiento de un individuo hacia los demás. La segunda se obtiene mediante manipulación experimental o un programa de intervención. Generalmente, es a través del proceso de ésta que los videojuegos pueden fomentar la empatía. Dependiendo del diseño del juego y la estrategia de implementación, pueden utilizar empatía tanto cognitiva como emocional.

Los juegos serios se consideran una herramienta viable para generar empatía, ya que "permiten a los jugadores ocupar los roles de otras personas de una manera única y

\footnotetext{
8 "[V]ideo games make claims about the world, which players can understand, evaluate, and deliberate" (Boghost, 2008, p. 119).

9 "[A]bility to see issues and events from the perspectives of people on the other side of a dispute" (Belman $y$ Flanagan, 2010, p. 6).

10 "[O]ne of the barriers to conflict resolution and reconciliation" (Malhotra y Liyanage, 2005, p. 908).
} 
envolvente". ${ }^{11}$ Una razón clave por la que los juegos son importantes para este proceso es que crean un espacio para el contacto virtual intergrupal que reduzca los prejuicios mediante la interacción. El contacto intergrupal en la generación de empatía se explora a continuación.

\section{Teoría de contacto intergrupal}

Fomentar la empatía intergrupal es un proceso que requiere que estos estén en contacto entre sí para crear un cierto nivel de confianza y reducir prejuicios negativos. Varios autores señalan la teoría del contacto intergrupal para explicar el proceso y el entorno en el que pueden ocurrir estas interacciones con videojuegos (Adachi, Hodson, y Hoffarth, 2015; Crisp y Turner, 2009; Hasler et al., 2014; Malhotra y Liyanage, 2005). La teoría del contacto intergrupal, o la teoría de la hipótesis del contacto, fue establecida por primera vez por Gordon Allport en 1954. Su argumento principal es que el contacto intergrupal puede reducir la ansiedad y los prejuicios hacia los grupos externos, al mismo tiempo que fomenta el conocimiento del grupo externo y aumenta la empatía (Adachi, Hodson, Willoughby, et al., 2015; Allport, 1954).

Los videojuegos proporcionan un espacio virtual para el contacto intergrupal ya que el contacto directo no siempre es viable debido a varias razones como la ubicación geográfica, estigma social, diferencias ideológicas y seguridad. Estudios han demostrado que cuando los grupos opuestos trabajaron juntos hacia una tarea de beneficio mutuo en un videojuego, el sesgo en el mundo real se redujo (Adachi, Hodson y Hoffarth, 2015; Adachi, Hodson, Willoughby, et al., 2015). Esto respalda el argumento acerca del potencial de algunos videojuegos como herramientas para facilitar el contacto entre grupos, ya que requieren que los jugadores trabajen hacia objetivos comunes.

Crisp y Turner (2009) entendieron las posibilidades de crear un espacio donde las interacciones no tenían que ser cara a cara y aún tener resultados positivos en la empatía intergrupal. A partir de la teoría de Allport desarrollaron la teoría de contacto intergrupal imaginado (CII) como un espacio alternativo de diálogo y cooperación. Según Crisp y Turner

El contacto intergrupal imaginado es la estimulación mental de una interacción social con un miembro o miembros de una categoría externa, [a través de la cual] una experiencia de contacto positivo estimulante mentalmente, activa conceptos normalmente asociados con interacciones exitosas con miembros de otros grupos. ${ }^{12}$

Cuando el CII es positivo, se incrementan las posibilidades de proyecciones positivas de los exogrupos y pueden conducir a la activación de procesos cognitivos y conductuales similares al contacto en persona, cuyos resultados pueden medirse por la reducción de ansiedad y de amenaza del estereotipo negativo que se tenía en primera instancia. Los niveles de ansiedad que surgen de la perspectiva de interactuar con un miembro de un grupo diferente se reducen cuando la interacción con "el otro" ha sido exitosa. La reducción de la ansiedad revela entonces una reducción del prejuicio negativo.

\footnotetext{
11 "[A]llow players to inhabit the roles of other people in a uniquely immersive way" (Belman \& Flanagan, 2010, p. 5).

12 "Imagined intergroup contact is the mental simulation of a social interaction with a member or members of an outgroup category. The basic idea is that mentally simulating a positive contact experience activates concepts normally associated with successful interactions with members of other groups" (Crisp y Turner, 2009, p. 234).
} 
Los videojuegos son una herramienta única para crear un espacio virtual para el CII debido a la oportunidad de enseñanza y aprendizaje activos cuando la situación no permita la interacción en persona. Además, la tecnología de los juegos digitales ofrece ventajas únicas como la capacidad de construir un contexto para los procesos de toma de decisiones en un entorno controlado. La complejidad de la vida real se puede capturar a través de videojuegos que brindan una experiencia de aprendizaje más profunda a través del CII y construyen nociones de empatía más sólidas. Además, esta tecnología puede reducir costos y unir a las personas cuando de otra manera no sería posible (Cuhadar y Kampf, 2014).

Los videojuegos serios ofrecen al usuario un conjunto de características que los convierten en una herramienta adecuada para fomentar las relaciones a través del CII. Primero, proporcionan un tercer espacio en el cual "crear mundos y realidades delimitados", ${ }^{13}$ ofreciendo al usuario un espacio para la simulación en un entorno seguro y sin consecuencias serias. Los terceros espacios se consideran los lugares de encuentro fuera del hogar y el trabajo donde las personas se reúnen con otras que comparten intereses similares y están expuestas a otras personas que tienen diferentes sentidos del mundo. Con la globalización facilitando el crecimiento de las redes transnacionales, se ha ampliado el concepto, incluyendo ahora el ciberespacio. Este tercer espacio aumenta la capacidad para difundir mensajes y vincular grupos con ideas afines a través de fronteras geopolíticas y culturales (Hjarvard, 2011). Al mismo tiempo, brinda la oportunidad de CII entre quienes no comparten los mismos puntos de vista. Por lo tanto, el tercer espacio virtual creado por los videojuegos serios, conocido como gameworld, brinda la oportunidad de explorar, aprender y experimentar la resolución de conflictos y desarrollar empatía (Cheong et al., 2011).

Segundo, los videojuegos en general, y los videojuegos serios en particular, desarrollan un conjunto de habilidades a través de la práctica y la internalización que son "relevantes para el comportamiento en mundo real y pueden transferirse al conocimiento y comportamientos"14 de la vida diaria. Varios autores han explorado las implicaciones en el mundo material de las nociones aprendidas en videojuegos y la opinión general es que esas habilidades brindan una nueva oportunidad para el aprendizaje cognitivo en un entorno de estrés reducido (Adachi et al., 2015; Cheong et al., 2011; Hill et al., 2009). Algunos estudios sobre empatía sugieren que existe una relación directa entre la empatía y el comportamiento prosocial (Belman y Flanagan, 2010), situando el avance de la práctica través de los videojuegos como un proceso que desarrollará actitudes que podrían traducirse en empatía emocional y cambio de comportamiento en el mundo real.

Sin embargo, los diseñadores de videojuegos deben ser conscientes de que sus propios prejuicios pueden ser programados en la narrativa o reglas del juego y que la estrategia de distribución del juego también puede afectar el propósito educativo. Existe una reproducción problemática del pensamiento colonial en los videojuegos —en sus aspectos narrativos y lúdicos - que refuerza dinámicas de poder y patrones de creencias que sustentan el discurso dominante de la modernización como el desarrollo económico capitalista. En la siguiente sección, se explora la relación entre el cambio social y los videojuegos serios utilizando como

\footnotetext{
13 "[C]reate bounded worlds and realities" (Cheong et al., 2011, p. 16).

14 "[R]elevant to real world behavior and can be transferred to real world knowledge and behaviors" (Cheong et al., 2011, p. 16).
} 
caso de estudio el juego EVOKE, creado por encargo del Banco Mundial. El enfoque principal de esta sección es la crítica del discurso colonial incorporado en el juego.

\section{EVOKE}

El trabajo y las perspectivas contemporáneas sobre edutainment han ampliado su alcance para incluir formas de comunicación interactivas (Storey y Sood, 2013). Los videojuegos conforman un entorno en el que puede tener lugar el aprendizaje situado que sugiere que el cerebro "aprende mejor al 'hacer' activamente en un entorno significativo". ${ }^{15}$ Bajo esta noción, los videojuegos pueden influir en el proceso de toma de perspectiva necesario para el desarrollo exitoso de la empatía y el cambio social ya que cuando los jugadores se adentran al mundo del juego, son responsables de sus elecciones en un entorno seguro. En esta sección, se analizan los esfuerzos y resultados del Banco Mundial en el diseño del juego $E V O K E$ para el cambio social.

$E V O K E$ es un videojuego serio de rol multijugador masivo en línea que busca incentivar a los jóvenes a que se conviertan en innovadores sociales creando soluciones para los desafíos globales mientras construyen redes internacionales (Gaible y Dabla, 2010). Fue concebido y coordinado por Robert Hawkings por encargo del Banco Mundial (BM) y se desarrolló en un esfuerzo por ayudar a los jóvenes a comprender los desafíos globales del nuevo siglo como el desplazamiento, hambruna, pobreza y escasez de agua mientras desarrollan habilidades para la colaboración, creatividad y reflexión crítica.

Evoke ha tenido cinco iteraciones, pero para los propósitos de este documento, el enfoque será en la primera: Urgent EVOKE. Diseñado y cocreado por Jane McGonigal, fue lanzado en inglés en 2010 como un programa de 10 semanas para "empoderar a los jóvenes en todo el mundo, y especialmente en África, para comenzar a resolver problemas sociales urgentes". ${ }^{16}$ El objetivo era ayudar a los jóvenes de 15 a 24 años a comprender las “experiencias vividas por las personas en su comunidad, brindándoles un modelo educativo en el que puedan conocer el ciclo de innovación social a través de la investigación y de la experiencia de primera mano en el campo". ${ }^{17}$ El juego se desarrolló respondiendo a una solicitud del gobierno sudafricano "para involucrar a los jóvenes en la innovación social y el compromiso cívico"18 con sus comunidades y se preveía una participación mayoritaria de los jóvenes del África Subsahariana (ASS). El arco narrativo del juego sigue a un grupo de talentosos innovadores sociales africanos en el año 2020 que abordan desafíos globales como el acceso a agua limpia, energía renovable y escasez de alimentos (Freeman y Hawkins, 2016).

$E V O K E$ fue diseñado como una novela gráfica y un juego digital de innovación social con actividades basadas en la comunidad que proponían un desafío semanal, diez desafíos en total para completar el programa, para que los jugadores crearan lazos con sus compañeros de juego en línea y con miembros de su comunidad fuera de ella para

\footnotetext{
15 "[L] earns better by actively "doing" in a meaningful setting" (Darvasi, 2016, p. 6).

16 "[E]mpower young people over the world, and especially in Africa, to start solving urgent social problems" (Hawkins, 2010, s/p).

17 "[L]ived experiences of people in the community by providing an educational model in which they can learn about the social innovation cycle through research and from first-hand experience in the field" (Freeman y Hawkins, 2016, p. $1)$.

18 “[T] get young people involved in social innovation and civic engagement" (Freeman y Hawkins, 2016, p. 2).
} 
desarrollar estrategias para cambiar el mundo (Freeman y Hawkins, 2016). Al décimo desafío, Evokation, los jugadores diseñaban un proyecto social en equipo y lo modelaban en el mundo real, subiendo la evidencia a la plataforma del juego. El juego impulsaba a los jugadores a hacerse responsables de su aprendizaje en el mundo digital poniéndolo en práctica en el mundo real. Quienes lograban completar los diez desafíos recibían un certificado de innovador social por parte del BM.

Si bien el potencial de EVOKE para proporcionar un tercer espacio digital en el que los jugadores pudieran crear redes transnacionales era fuerte, la realidad mostró fallas en la concepción, gestión y alcance del proyecto. El juego registró a 19,386 personas de todo el mundo, pero no logró llegar a la población objetivo en el continente africano (Waddington, 2013). Del total de participantes registrados, "sólo 1,529 eran del ASS: 1,010 de estos jugadores eran de Sudáfrica, mientras que otros 519 eran del resto de los países del ASS combinados [...] en comparación con los 9,577 jugadores de Estados Unidos, o los 1,672 de Canadá". ${ }^{19}$ Del número total de jugadores registrados, 6,618 (34\%) completaron al menos una misión, 142 personas $(0.73 \%)$ completaron las diez misiones y 73 personas $(0.37 \%)$ completaron el desafío final Evokation subiendo su proyecto a la plataforma del juego (Gaible y Dabla, 2010). Estas cifras muestran varios problemas con la aplicación del juego.

EVOKE se publicitó solo en Sudáfrica a través de una campaña publicitaria que le brindó visibilidad con el público general y al mismo tiempo, formaba relaciones con las escuelas secundarias y universidades para crear un grupo consistente de jugadores. El resultado de la campaña se ve en la gran cantidad de jugadores de Sudáfrica en comparación con los países vecinos, pero las aulas evidenciaron las brechas tecnológicas y sociales del país: el acceso a Internet en diversas partes de Sudáfrica no era confiable lo que dificultaba el juego. Probablemente más importante en este análisis, "cuestiones de género, habilidades lingüísticas y clase social influyeron en quiénes llegaron a jugar". ${ }^{20}$ A pesar de esto y de que Sudáfrica no constituyó la nacionalidad con más jugadores registrados, eran los más comprometidos con el juego, pasaban más tiempo en su sitio web y completaron $40 \%$ más misiones que sus pares en otros países al publicar más blogs, fotos y videos como evidencia del cumplimiento de las misiones (Gaible y Dabla, 2010).

Según las respuestas a la encuesta oficial del juego, después de su participación los jugadores subsaharianos cambiaron la forma en que pensaban sobre problemáticas sociales y su resolución, y la confianza en sí mismos y sentido de agencia con respecto a hacer un cambio en el mundo aumentó (Gaible y Dabla, 2010). A primera vista, estos resultados logran el objetivo de incrementar las habilidades socioemocionales como la empatía y el compromiso cívico. Sin embargo, la participación en $E V O K E$ requería acceso confiable a Internet, equipos y habilidades tecnológicas adicionales para navegar por la interfaz del juego publicar evidencia de los desafíos y blogs en la plataforma (Fisher, 2016), conocimiento de inglés en el que se presentó el juego y un grado de habilidad con y acceso a las plataformas de redes sociales (Gaible y Dabla, 2010). Estas condiciones no están disponibles para la mayoría de las personas en los países subsaharianos, lo que limita la participación a un segmento de la población privilegiado y educado, como estudiantes universitarios y jóvenes profesionales.

\footnotetext{
19 " [O]nly 1,529 were from SSA: 1,010 of these players were from South Africa, while other 519 were from the rest of the SSA countries combined [...] compared to the 9,577 players in the United States, or the 1,672 in Canada" (Fisher, 2016, p. 36).

20 "[I]ssues of gender, language skills, and social class affected who got to play" (Fisher, 2016, p. 36).
} 
En ese escenario, el objetivo de EVOKE de desarrollar habilidades para los problemas globales del siglo XXI es efectivo solo entre la élite. Si bien EVOKE fue diseñado para empoderar a los jóvenes de todo el mundo, pero específicamente en África Subsahariana, son ellos quienes tienen más barreras que les impiden adquirir estas habilidades debido a su contexto. En última instancia, esto es un obstáculo para la reconfiguración de las estructuras de poder y perpetúa una ideología neoliberal que enfatiza "las habilidades de mercado y el ingenio individual como clave para resolver problemas sociales amplios" (Fisher, 2016, p. 36). ${ }^{21}$ EVOKE es un juego que entiende el desarrollo social y económico como la expansión y el apoyo del mercado económico y es responsabilidad del individuo adquirir las habilidades necesarias para participar en él. Al considerar la modernidad como un proceso engendrado por la colonialidad (Mignolo y Walsh, 2018), los participantes están siendo instruidos en un modelo que fortalece la matriz colonial del poder en los niveles de conocimiento, autoridad política y economía al restringir las habilidades deseables al desarrollo económico, situar las instituciones coloniales como proveedoras de conocimiento y el capitalismo como el único sistema económico deseado, respectivamente.

La empatía es una de las habilidades que se espera que el jugador desarrolle a lo largo del juego, pero al hacerlo, EVOKE enfatiza el valor de la innovación tecnológica, el compromiso con la economía global y la dependencia en los sistemas tecnológicos. Es comprensible que el juego apoye la agenda del Banco Mundial, pero también es importante considerar por qué estas habilidades se presentan como una forma de crear empatía global y cambio social. Esto alude al concepto de imperialismo mediático, que ve en las tecnologías digitales la posibilidad infinita de la modernidad sin criticar el papel que tienen en la continuación de la retórica y la opresión colonial (Boyd-Barrett, 2015). Sumado a esto, la idealización de la comunidad global que surgiría gracias a la tecnología digital como los videojuegos, dejó de lado las limitaciones económicas y educativas que aparecen ante la disparidad conectiva. Paralelamente a "la misión civilizadora de los colonialismos anteriores, la retórica utópica de los medios electrónicos ocluye el proyecto práctico de crear nuevos mercados y fuerzas de trabajo para las empresas capitalistas" (Fernández, 1999, p. 60). ${ }^{22}$

Es importante desafiar el significado de la empatía y su papel en la preservación de las estructuras de poder. La empatía se considera un componente clave para sensibilizar a quienes participan en el diálogo intercultural, como lo proponía EVOKE. Se busca crear una conexión emocional o cognitiva que reduzca el estrés hacia lo desconocido o las percepciones negativas previamente formadas y por ende permita proyecciones positivas (Bachen et al., 2012; Belman y Flanagan, 2010; Burak y Parker, 2017; Calloway-Thomas, 2010; Cowan y Arsenault, 2008; Finn, 2003; Shin y Ahn, 2013). Pero un problema que surge de esa noción es que es condescendiente y no reconoce las estructuras de poder que llevaron a la otredad en primer lugar.

Pedwell (2016) señala el llamado continuo de los líderes mundiales para "desarrollar actitudes y capacidades más empáticas como un medio para crear una sociedad global basada en un mayor respeto, cooperación e igualdad" (p. 2) ${ }^{23}$ En este escenario, la empatía se presenta

\footnotetext{
21 "[M]arket skills and individual resourcefulness as key to solving broad social problems" (Fisher, 2016, p. 36).

22 "[T] he civilizing mission in previous colonialisms, utopian rhetoric of electronic media occlude the practical project of creating new markets and work forces for capitalist enterprises" (Fernández, 1999, p. 60).

23 "[D]evelop more empathetic attitudes and capacities as a means to create a global society built on greater respect, cooperation and equality" (Pedwell, 2016, p. 2).
} 
como una solución afectiva para fortalecer las relaciones transculturales y promover la justicia social. Pide a las personas que sean compasivas con las circunstancias de los demás para neutralizar los sentimientos de miedo y rechazo, pero no pide una reflexión sobre la forma en que se transforma y se traduce en diferentes grupos sociales y culturales y sus vínculos con las relaciones transnacionales de poder.

Varios autores (Gunew, 2009; Pedwell, 2016; Sandoval, 2003; Smith, 2012) enfatizan que cuando la empatía se entiende como un sentimiento compartido por ambas partes del espectro (empatizador-empatizado), la relación de poder inherente pone a los sujetos privilegiados en una posición en la que no solo se apropian superficialmente de la desventaja del subalterno, sino que también se vuelven incapaces de ver su complicidad con los sistemas que los colocan en un punto de ventaja sobre los demás. La empatía se vuelve pasiva y satisface una agenda multicultural que mantiene las estructuras de poder y no conduce efectivamente a su transformación o a la justicia social (Boler, 1999).

Ante esto, se hace un llamado a entender la empatía como un compromiso radical donde el empatizador/privilegiado pregunta quién se beneficia de la empatía e inicia un proceso autorreflexivo de las relaciones de poder que llevaron a ese posicionamiento. Además, la empatía debe situar al empatizador en un lugar para "reconocerse a sí mismo como implicado en las fuerzas sociales que crean el clima de obstáculos que el otro debe enfrentar". 24 Para ello, Pedwell (2016) sugiere empatías alternativas que se "expresan desde los márgenes de los imaginarios sociales poscoloniales dominantes [y] pueden diferir de las principales movilizaciones liberales y neoliberales, así como [...] desorganizar o reconfigurar sus lógicas afectivas". ${ }^{25}$

Por otro lado, la problemática orientalización de los países subsaharianos por parte de $E V O K E$ refuerza un discurso neocolonial en el que el estado postcolonial, en este caso los países subsaharianos y principalmente Sudáfrica, permanece en una situación de dependencia económica con sus contrapartes colonizadoras personificadas el Banco Mundial (Young, 2001). La interpretación del BM de "habilidades deseadas" para el siglo XXI sigue un enfoque eurocéntrico, adaptado para la teorización intelectual, que funcionan como herramientas hegemónicas que se aplican universalmente, asumiendo que la experiencia europea es lo que define al ser humano (Oyewumi, 1997).

La creación de una distinción entre el yo y el otro ha sido utilizada como método de control por sociedades coloniales, creando estructuras de poder basadas en diferencias físicas y culturales (Quijano, 2001). En el caso de los videojuegos, estos presentan una plataforma interesante para abordar la identidad y la alteridad ya que existe la posibilidad de desafiar estas concepciones tradicionales. El mundo virtual se construye, deconstruye y reconstruye constantemente de acuerdo a un conjunto de creencias llevadas a cabo por el programador y el jugador. Con respecto a la construcción de la identidad en línea, una discusión central es "el grado en que la existencia en línea está alienada de la interacción fuera de ella, y el grado en

\footnotetext{
24 "“[R] ecognize oneself as implicated in the social forces that create the climate of obstacles the other must confront"” (Boler, 1999, p. 166, en Pedwell, 2016, p. 7).

25 "[E]xpressed from the margins of dominant postcolonial social imaginaries might differ from mainstream liberal and neoliberal mobilisations, as well as how it might disrupt or refigure their affective logics (Pedwell, 2016, p. 15).
} 
que esta alienación es reconocida o no". ${ }^{26}$ Es decir, con las posibles identidades siendo determinadas por el programador, las que no están representadas son excluidas y borradas.

Finalmente, al implementar estas tecnologías, las organizaciones interesadas deben reevaluar cómo el mensaje y las habilidades que están tratando de desarrollar actúan como una "intervención sobre el terreno que refuerza un enfoque de desarrollo [social y económico] problemático en el que se abordan todos los elementos de la vida social, incluida la educación, son negociadas como parte del mercado económico". ${ }^{27}$ EVOKE se desarrolló con buenas intenciones, pero no reconoce el papel del colonialismo europeo en producir el desequilibrio económico de la zona en primer lugar. Por el lado positivo, está ocurriendo un proceso decolonial dentro del espacio de los videojuegos. La siguiente sección hace un repaso de estos esfuerzos y analiza los beneficios del análisis crítico en la implementación de estrategias por organizaciones internacionales como el BM.

\section{Decolonialidad en juego}

La decolonialidad aquí se entiende como el "ejercicio del poder dentro de la matriz colonial para socavar el mecanismo que lo mantiene en ese lugar" (Mignolo y Walsh, 2018, p. 114). ${ }^{28}$ Como señala Mignolo, "pensar de manera decolonial hizo posible ver la colonialidad, $y$ ver la colonialidad hizo posible materializar el pensamiento decolonial". ${ }^{29}$ En el contexto de los videojuegos, los jugadores tienen un papel clave en desafiar la retórica colonial infundida en la narrativa y la mecánica a través de sus acciones en el juego. Es el jugador, al crear historias alternas, quien también tiene un papel disruptivo a las concepciones coloniales del mundo del juego.

El videojuego ofrece un tercer espacio para establecer CII, pero este concepto puede extenderse a un tercer espacio para el Imaginario Decolonial donde los subalternos pueden escribir sus propias historias de nuevas formas que no están atadas a las historiografías eurocéntricas (Pérez, 1999). De esta manera, se pueden escribir narrativas alternas dentro del mundo del juego y el jugador puede responder a ellas (Mukherjee, 2016). El tercer espacio ofrece un sitio para el desarrollo de la empatía alternativa y ofrece "posibilidades simultáneas de subalternidad, protesta, elitismo y hegemonía; es la actualización por parte del jugador lo que da como resultado una comprensión y una experiencia más profundas de lo poscolonial". ${ }^{30}$ En otras palabras, podrían tener un "potencial retórico detrás del diseño de reglas de juego y la proyección de discursos éticos a través del diseño de experiencias de juego" 31 para cuestionar la empatía en el espacio lúdico.

\footnotetext{
26 "[T] $]$ he extent to which online existence is alienated from non-online interaction - and the extent to which this alienation is or is not recognized" (Langer, 2011, p. 87).

27 " $[\mathrm{O}] \mathrm{n}$-the-ground development intervention that reinforces a problematic development approach in which all elements of social life, including education, are taken up in the market" (Fisher, 2016, p. 37).

28 "[E]xercise of power within the colonial matrix to undermine the mechanism that keeps it in place requiring obeisance" (Mignolo y Walsh, 2018, p. 114).

29 "[T] hinking decolonially made it possible to see coloniality and seeing coloniality materialized decolonial thinking" (Mignolo y Walsh, 2018, p. 112).

30 "simultaneous possibilities of subalternity, protest, elitism, and hegemony; it is the actualization by the player that results in a deeper understanding and experience of the postcolonial (Mukherjee, 2016, p. 15).

31 "rhetorical potential behind the game rule design and the projection of ethical dis- courses through the design of game experiences" (Pérez Latorre, 2015, p. 417).
} 
Desde el punto de vista del desarrollador, hay una nueva ola de juegos independientes que están disputando el discurso colonial, la representación y la diversidad en la narrativa y mecánica de juegos. Se están asociando con comunidades indígenas, o en ocasiones son los mismos miembros de estas comunidades quienes están creando juegos basados en su cosmovisión y tradiciones, manteniendo la agencia narrativa. Never Alone y Mulaka son dos de los ejemplos más destacados de estas asociaciones.

Mulaka es un juego independiente desarrollado por el estudio mexicano Lienzo que explora la comunidad tarahumara (Joho, 2017). Edgar Serrano, el desarrollador del juego, creció en una comunidad tarahumara en el norte de México y quiere cambiar la forma en que la gente los percibe, dándoles a los tarahumaras un espacio para contar sus historias a través del juego. Se resalta la cultura de varias maneras: está narrado en rarámuri, la banda sonora usa instrumentos indígenas propios de la comunidad y las coloridas animaciones hacen referencia a su vestimenta y arte geométrico (Barasch, 2018). Mulaka es un juego de acción que sigue a un chamán tarahumara que lucha contra "la maldad que corrompe la tierra, mientras se basa en los poderes de los semidioses" (Lienzo, s/f). Las misiones tienen lugar en la Sierra Tarahumara y exploran los diferentes ecosistemas de la región. Como acciones decoloniales fuera del mundo virtual, una parte de las ganancias del juego se dona para fortalecer la cultura y preservar la Sierra Tarahumara (Lienzo, s/f).

Never Alone utiliza un enfoque similar. Es un juego desarrollado y publicado por Upper One Games y E-Line Media que se basa en la historia Kunuuksaayuka de la comunidad indígena Iñupiaq, en la que una niña de una sociedad nómada de Alaska emprende la misión de salvar a su comunidad de una tormenta de nieve catastrófica (Byrd, 2014). El objetivo de este proyecto es contar la historia oral de los nativos de Alaska para preservar sus historias tradicionales compartiéndolas dentro de su propia comunidad, especialmente las generaciones más jóvenes, y con personas de todo el mundo. En este esfuerzo por compartir su cultura desde su propia voz, los desarrolladores esperan crear conciencia sobre la importancia de preservar las tradiciones y cosmovisiones indígenas, así como el ecosistema del Ártico. Never Alone fue desarrollado por y con miembros de la comunidad quienes cuentan su historia en lugar de que personas ajenas lo hagan. Este juego proporciona una experiencia a los jugadores en la que se les invita a conectarse con percepciones del mundo distintas a las suyas (Peckham, 2014), las cuales se transmiten en la narrativa y mecánica.

Ambos ejemplos muestran cómo se puede operacionalizar la decolonialidad dentro de la matriz colonial que rige la industria del videojuego. Al trabajar desde adentro del sistema, estos juegos están impulsando una independencia epistemológica y mental de las nociones hacia sus culturas. Están recuperando sus idiomas y creando tramas a través de las cuales retoman control de la narrativa sobre sus culturas indígenas, tomando agencia de su conocimiento y vivencias. Al desvincularse del discurso eurocéntrico de sus historias, estos juegos están transformando la matriz colonial de poder de Mignolo y abriendo vías alternativas para fomentar empatías alternativas hacia diferentes formas de existencia.

A través de juegos como Never Alone y Mulaka, las sociedades oprimidas están ejecutando una mirada de oposición a la producción - y los productores - culturales que perpetúan una estructura de poder sobre ellas (hooks, 1992). Como ha expresado hooks, la mirada de oposición, se convierte en una forma de resistencia que se puede realizar después de reconocer cómo el colonizador ha inferido en la identidad de quienes la hacen. Los desarrolladores de los juegos independientes mencionados, después de reconocer el papel del 
colonizador, están brindando una posibilidad de agencia a sus comunidades a través del trabajo cooperativo de diseño y desarrollo del juego. En estos videojuegos, las comunidades Iñupiaq y Tarahumara ahora están creando un espacio para ellos mismos en el cual demoler los estereotipos nocivos que todavía los retratan en la actualidad. Desde la perspectiva de los estudios de juegos, Never Alone y Mulaka son ejemplos de que los videojuegos serios pueden ser entretenidos, tener éxito en su propósito informativo y ofrecer un espacio decolonial para la crítica de la otredad.

\section{Conclusiones}

A lo largo de este artículo, se estableció el contexto para argumentar que los juegos digitales son una herramienta que ofrece una perspectiva única sobre la construcción de percepciones empáticas entre los individuos. La empatía es un elemento clave para el cambio social y los videojuegos pueden proporcionar un tercer espacio digital en el que la colaboración intergrupal se puede llevar a cabo. Por otro lado, es importante tener una comprensión clara de cuál será el objetivo del videojuego ya que para obtener resultados en el ámbito del cambio social se requiere negociar con múltiples intereses. Los juegos digitales por sí solos no son una herramienta que traerá ese cambio. El contexto actual de globalización exige la cooperación entre diferentes campos y el uso de múltiples recursos para lograr un impacto significativo en el mundo.

Es urgente una crítica consciente del proceso en el que las organizaciones internacionales y los Estados entienden la modernidad para evitar la recreación de estructuras coloniales. Asumir que una postura neoliberal y la globalización son beneficiosas para todos quienes están jugando, especialmente en países en el Sur Global, plantea intenciones problemáticas, así como la suposición de que estos elementos son necesarios para que suceda el llamado 'desarrollo' (Fisher, 2016). Las instituciones que confían a los desarrolladores el diseño de juegos deberán revisar sus objetivos finales y tener en cuenta sus propios prejuicios. En ocasiones, los juegos promoverán la idea de desarrollo, paz o reconciliación desde una perspectiva paralela a los sistemas capitalistas que no son pertinentes para todas las sociedades y puede ignorar el rol que el sistema tiene en el incremento de la desigualdad en diferentes contextos. Si la decolonialidad no puede ocurrir en proyectos liderados por Estados sino a través de individuos que organizan sus propias historias locales (Mignolo y Walsh, 2018), entonces los videojuegos como Mulaka y Never Alone pueden convertirse en espacios donde estas conversaciones tengan lugar, abriendo el camino a la comunicación intercultural y al intercambio de experiencias y significados. Como se señaló anteriormente, disminuir la ansiedad de interactuar con una cultura desconocida da como resultado un aumento de los niveles de empatía y una reducción paulatina de los estereotipos negativos. Por lo tanto, las estrategias de cambio social que se centren en el desarrollo de empatía a través de videojuegos serios deberán repensar el rol de las organizaciones internacionales como el Banco Mundial y dar más visibilidad a los juegos independientes como Mulaka y Never Alone, donde sean las mismas comunidades quienes tengan agencia sobre su narrativa y poder de toma de decisiones.

Finalmente, es importante reconocer que los juegos digitales requieren un conjunto de equipos tecnológicos que no está al alcance de todos. Si bien los juegos en línea pueden ser más accesibles siempre que haya una conexión a Internet y un dispositivo adecuado, los juegos que requieren una consola para operar impondrán ciertas barreras de acceso dada la necesidad 
de solvencia económica, lo que dejará a algunos fuera del alcance de dichas estrategias. Los grupos de interés que planean utilizar prácticas basadas en videojuegos en zonas de bajos recursos y/o baja conectividad, deben tenerlo en cuenta a la hora de presupuestar la aplicación del programa, así como pensar en su continuación en el futuro. Sin embargo, los juegos digitales abarcan una amplia gama de puntos de venta y se pueden diseñar juegos más simples para adaptarse a diferentes accesos tecnológicos.

\section{Referencias}

Adachi, P. J. C., Hodson, G., y Hoffarth, M. R. (2015). Video game play and intergroup relations: Real world implications for prejudice and discrimination. Aggression and Violent Behavior, 25, 227-236. https://doi.org/10.1016/j.avb.2015.09.008

Adachi, P. J. C., Hodson, G., Willoughby, T., y Zanette, S. (2015). Brothers and sisters in arms: Intergroup cooperation in a violent shooter game can reduce intergroup bias. Psychology of Violence, 5(4), 455-462. https://doi.org/10.1037/a0037407

Allport, G. W. (1954). The nature of prejudice. Addison-Wesley Publishing Company.

Bachen, C. M., Hernández-Ramos, P. F., y Raphael, C. (2012). Simulating REAL LIVES: Promoting global empathy and interest in learning through simulation games. Simulation y Gaming, 43(4), 437-460. https://doi.org/10.1177/1046878111432108

Barasch, A. (2018, abril 10). 'Mulaka': Struggle of exploring Tarahumara Lore in a video game. Variety. http://variety.com/2018/gaming/features/mulaka-mexico-lienzodeveloper-1202749723/

Belman, J., y Flanagan, M. (2010). Designing games to foster empathy. International Journal of Cognitive Technology, 15(1), 5-15.

Bogost, I. (2006). Videogames and ideological frames. Popular Communication, 4(3), 165183. https://doi.org/10.1207/s15405710pc0403_2

Bogost, I. (2008). The rhetoric of video games. En K. Salen (Ed.), The Ecology of Games: Connecting Youth, Games, and Learning (pp. 117-140). The MIT Press.

Bogost, I. (2010). Persuasive games: The expressive power of videogames. The MIT Press.

Boler, M. (1999). Feeling power: Emotions and education. Taylor y Francis Group.

Boyd-Barrett, O. (2015). Media imperialism. SAGE Publications.

Burak, A., y Parker, L. (2017). Power play: How video games can save the world. St. Martin's Press.

Byrd, C. (2014, diciembre 29). In "Never Alone" Native Alaskans explore the future of oral tradition. The Washington Post. https:/www.washingtonpost.com/news/comicriffs/wp/2014/12/29/never-alone-review-native-alaskans-explore-the-future-of-oraltradition/

Calloway-Thomas, C. (2010). Empathy in the global world: An intercultural perspective. SAGE Publications.

Campbell, H. A., Wagner, R., Luft, S., Gregory, R., Grieve, G. P., y Zeiler, X. (2016). Gaming religionworlds: Why religious studies should pay attention to religion in gaming. Journal of the American Academy of Religion, 84(3), 641-664. https://doi.org/10.1093/jaarel/lfv091. 
Cheong, Y.-G., Khaled, R., Grappiolo, C., Campos, J., Martinho, C., Ingram, G., Paiva, A., y Yannakakis, G. (2011). A computational approach towards conflict resolution for serious games. Proceedings of the 6th International Conference on Foundations of Digital Games, 15-22.

Cowan, G., y Arsenault, A. (2008). Moving from monologue to dialogue to collaboration: The three layers of public diplomacy. The ANNALS of the American Academy of Political and Social Science, 616(1), 10-30. https://doi.org/10.1177/0002716207311863.

Crisp, R. J., y Turner, R. N. (2009). Can imagined interactions produce positive perceptions?: Reducing prejudice through simulated social contact. American Psychologist, 64(4), 231-240. https://doi.org/10.1037/a0014718

Cuhadar, E., y Kampf, R. (2014). Learning about conflict and negotiations through computer simulations: The case of PeaceMaker. International Studies Perspectives, 15(4), 509524. https://doi.org/10.1111/insp.12076.

Darvasi, P. (2016). Empathy, perspective, and complicity: How digital games can support peace education and conflict resolution. En M. Faetanini y R. Tankha (Eds.), Social inclusion of internal migrants in India: Internal migration in India initiative. UNESCO.

Fernández, M. (1999). Postcolonial media theory. Art Journal, 58(3), 58-73. https://doi.org/10.2307/777861.

Finn, H. K. (2003). The case for cultural diplomacy: Engaging foreign audiences. Foreign Affairs, 82(6), 15-20. https://doi.org/10.2307/20033753.

Fisher, J. (2016). Toward a political economic framework for analyzing digital development games: A case study of three games for Africa. Communication, Culture y Critique, 9(1), 30-48. https://doi.org/10.1111/cccr.12128.

Foucault, M., y Gordon, C. (1980). Power/knowledge: Selected interviews and other writings, 1972-1977. Pantheon Books.

Freeman, B., y Hawkins, R. (2016). EVOKE-Developing skills in youth to solve the world's most complex problems: The social innovators' framework. The World Bank. https://bit.ly/3cyEle5.

Gaible, E., y Dabla, A. (2010). Project evaluation: EVOKE. Natoma Group. http://siteresources.worldbank.org/EDUCATION/Resources/ProjectEVOKEevaluation-final-16oct11.pdf.

Gunew, S. (2009). Subaltern empathy: Beyond European categories in affect theory. Concentric: Literary and Cultural Studies, 35(1), 11-30.

Hasler, B. S., Hirschberger, G., Shani-Sherman, T., y Friedman, D. A. (2014). Virtual peacemakers: Mimicry increases empathy in simulated contact with virtual outgroup members. Cyberpsychology, Behavior, and Social Networking, 17(12), 766-771. https://doi.org/10.1089/cyber.2014.0213.

Hawkins, R. (2010, febrero 11). EVOKE: A crash course in changing the world. Edutech. https://bit.ly/3cxujKg.

Hill, J. R., Song, L., y West, R. E. (2009). Social learning theory and web-based learning environments: A review of research and discussion of implications. American Journal of Distance Education, 23(2), 88-103. https://doi.org/10.1080/08923640902857713.

Hjarvard, S. (2008). The mediatization of society. Nordicom Review, 29(2), 105-134. 
Hjarvard, S. (2011). The mediatisation of religion: Theorising religion, media and social change. Culture and Religion, 12(2), 119-135. https://doi.org/10.1080/14755610.2011.579719.

hooks, bell. (1992). Black looks: Race and representation. South End. https://doi.org/10.1007/978-1-137-08886-4_12.

Joho, J. (2017, septiembre 29). An indie game that aims to preserve an Indigenous culture and its mythology. Mashable. https://bit.ly/3kT8FEM.

Langer, J. (2011). Race, culture, identity and alien/nation. En Postcolonialism and Science Fiction (pp. 81-106). Palgrave Macmillan UK. https://doi.org/10.1057/9780230356054.

Lienzo. (s/f). Mulaka. Mulaka. http://www.lienzo.mx/mulaka/.

Malhotra, D., y Liyanage, S. (2005). Long-term effects of peace workshops in protracted conflicts. Journal of Conflict Resolution, 49(6), 908-924.

Marsh, T. (2011). Serious games continuum: Between games for purpose and experiential environments for purpose. Entertainment Computing, 2(2), 61-68. https://doi.org/10.1016/j.entcom.2010.12.004.

Mignolo, W. D., y Walsh, E. C. (2018). On decoloniality: Concepts, analytics, praxis. Duke University Press.

Millington, B. (2016). Video games and the political and cultural economies of health$\begin{array}{llll}\text { entertainment. } & \text { Leisure } & \text { Studies, } & \text { 35(6), }\end{array}$ https://doi.org/10.1080/02614367.2014.986511.

Mills, S. (2013). Gender and colonial space. Manchester University Press.

Mukherjee, S. (2016). Playing subaltern: Video games and postcolonialism. Games and Culture, 13(5), 504-520. https://doi.org/10.1177/1555412015627258.

Nakamura, L. (2002). Cybertypes: Race, Ethnicity, and Identity on the Internet. Routledge.

Oyewumi, O. (1997). Visualizing the body: Western theories and African subjects. En O. Oyewumi, Invention of Women: Making An African Sense Of Western Gender Discourses (pp. 1-30). University of Minnesota Press. https://doi.org/10.1007/978-1137-09009-6_1.

Peckham, M. (2014, noviembre 21). Never Alone is a harrowing journey into the folklore of Alaska Natives. WIRED. https://www.wired.com/2014/11/never-alone-review/.

Pedwell, C. (2016). Decolonising empathy: Thinking affect transnationally. Samyukta: A Journal of Women's Studies, XVI(1), 27-49.

Pérez, E. (1999). The decolonial imaginary: Writing Chicanas into history. Indiana University Press.

Pérez Latorre, O. (2015). The social discourse of video games analysis model and case study: GTA IV. Games and Culture, 10(5), 415-437. https://doi.org/10.1177/1555412014565639.

Quijano, A. (2001). Colonialidad del poder, eurocentrismo y América Latina. En W. D. Mignolo (Ed.), Capitalismo y geopolítica del conocimiento: El eurocentrismo y la filosofía de la liberación en el debate intelectual contemporáneo (pp. 201-246). Ediciones del Signo.

Sandoval, C. (2003). Methodology of the oppressed. University of Minnesota Press. 
Sanford, K., Starr, L. J., Merkel, L., y Bonsor Kurki, S. (2015). Serious games: Video games for good? E-Learning and Digital Media, 12(1), 90-106. https://doi.org/10.1177/2042753014558380.

Sheldon, M. (2008). Continuing the colonial process through video games. MAI Review, 2, 1-5.

Shin, D.-H., y Ahn, D. (2013). Associations between game use and cognitive empathy: A cross-generational study. Cyberpsychology, Behavior, and Social Networking, 16(8), 599-603. https://doi.org/10.1089/cyber.2012.0639.

Singhal, A. (2013). Introduction: Fairy tales to digital games: The rising tide of entertainment education. Critical Arts, 27(1), 1-8. https://doi.org/10.1080/02560046.2013.766971.

Singhal, A., y Rogers, E. M. (1999). Entertainment-education: A communication strategy for social change. L. Erlbaum Associates.

Smith, L. T. (2012). Decolonizing methodologies: Research and Indigenous Peoples (2nd ed.). Zed Books.

Storey, D., y Sood, S. (2013). Increasing equity, affirming the power of narrative and expanding dialogue: The evolution of entertainment education over two decades. Critical Arts, 27(1), 9-35. https://doi.org/10.1080/02560046.2013.767015.

The World Bank. EVOKE - An online alternate reality game supporting social innovation among young people around the world. (s.f.). https://bit.ly/2Z5NoA5.

Waddington, D. (2013). A parallel world for the World Bank: A case study of Urgent: Evoke, an educational alternate reality game. International Journal of Technologies in Higher Education, 10(3), 42-56. https://doi.org/10.7202/1035578ar.

Wang, H., y Singhal, A. (2009). Entertainment-education through digital games. En U. Ritterfeld, M. J. Cody, y P. Vorderer (Eds.), Serious games: mechanisms and effects (pp. 271-292). Routledge.

Young, R. (2001). Postcolonialism: An historical introduction. Blackwell Publishers. 